\title{
Structures, Stabilities, Reactivities, and (Hyper)Polarizabilities of Small Gold Clusters
}

\author{
Francisco E. Jorge $e^{*, a, b}$ and Alexsandro S. Santos ${ }^{a}$ \\ ${ }^{a}$ Unidade Acadêmica de Física, Universidade Federal de Campina Grande, \\ 58429-900 Campina Grande-PB, Brazil \\ ${ }^{b}$ Departamento de Física, Universidade Federal do Espírito Santo, 29060-900 Vitória-ES, Brazil
}

\begin{abstract}
At the Douglas-Kroll-Hess level, the B3PW91 hybrid functional along with relativistic all-electron basis sets are used to evaluate geometric parameters, binding energies, vertical ionization potentials and electron affinities, and HOMO-LUMO (highest occupied molecular orbital-lowest unoccupied molecular orbital) energy gaps of the small golden clusters $\left(\mathrm{Au}_{\mathrm{n}}, \mathrm{n} \leq 8\right)$. The so expected odd-even oscillations of the experimental ionization potentials and electron affinities are confirmed in this work and the $\mathrm{Au}_{7}$ cluster is predicted to be the most reactive cluster. Using the optimized geometries, DKH2 static mean dipole polarizability and polarizability anisotropy are also computed. From $n \geq 2$, the mean dipole polarizabilities per atom present an odd-even oscillatory characteristic, whereas the polarizability anisotropies increase with the cluster size. At the non-relativistic level, the second hyperpolarizabilities are calculated. It is the first time that hyperpolarizabilities of gold clusters are reported. Comparisons with theoretical results obtained previously for the copper and silver clusters at the same level of theory are made.
\end{abstract}

Keywords: B3PW91 functional, all-electron basis sets, DKH2 calculations, gold clusters, (hyper)polarizabilities

\section{Introduction}

Atomic cluster is formed by an assembling of a few or hundreds of atoms. It is necessary to understand how the cluster properties vary with the size, to clarify how they evolve in the direction of the bulk properties. Metal clusters have received special attention in the literature due to their unusual characteristics, properties, and applications to build new electronic devices. ${ }^{1}$ Theoretical ${ }^{2-11}$ and experimental ${ }^{12-19}$ studies about metal clusters have been carried out for about thirty three years. Among the metal clusters, there is an increasing interest ${ }^{20-32}$ in the noble metals $(\mathrm{Cu}, \mathrm{Ag}$, and $\mathrm{Au})$ because they can be considered as a connection between the alkali metals and the transition metals. We recall that their electronic configurations are $\mathrm{nd}^{10}(\mathrm{n}+1) \mathrm{s}^{1}$, with $\mathrm{n}=3,4$, and 5, respectively to $\mathrm{Cu}, \mathrm{Ag}$ and $\mathrm{Au}$. It has been verified that in general the gold clusters and compounds present large relativistic effects when compared to those of the copper and silver ones. ${ }^{33,34}$ We would like to cite the experiments about vertical ionization potentials and electron affinities performed by some research groups, ${ }^{28,35-39}$ where the results

*e-mail: jorge@cce.ufes.br of all studied gold clusters surpass those of the copper and silver ones (see Figure 2 of Wesendrup et al..). ${ }^{29}$ As a consequence of this finding, the properties and surfaces of the gold clusters will be differently affected. Thus, a study about the variation of such properties with the cluster size will be interesting. In this article, the vertical ionization potentials and electron affinities of gold clusters with up to 8 atoms as well as binding energies, highest occupied molecular orbital-lowest unoccupied molecular orbital (HOMO-LUMO) energy gaps, and polarizabilities will be compared with the corresponding ones obtained previously for the copper ${ }^{40}$ and silver ${ }^{41}$ clusters at the same level of theory. This comparison will provide a better understanding of how much the relativistic effects affect the properties of the noble metal clusters.

Most theoretical works were principally focused on the structures of gold clusters. After the pioneer work of Haruta et al. ${ }^{42-44}$ showing catalytic activity of gold nanoparticles, things have changed. It was shown that these particles can activate the oxidation of hydrogen dimer or carbon monoxide at low temperature. ${ }^{44,45}$

As polarizability is sensitive to the number of electrons and the shape of the cluster, it is employed in understanding 
the electronic structures of them. Its magnitude is associated with the electronic charge distortion suffered by a cluster due to an external electric field. In this work, to determine the most reactive gold clusters, the HOMO-LUMO energy gap along with the static mean dipole polarizability per atom are used, whereas to discuss the structures of the clusters, the polarizability anisotropy is used. The second hyperpolarizability is calculated as well. For the gold clusters, it is the first time that results of the latter property are reported in the literature.

Gold is a heavy metal. Therefore, it is needed to take into account simultaneously both the relativistic effects and electron correlation corrections in the calculations of gold cluster electronic structures. As the central processing unit (CPU) time enhances rapidly with the cluster size, effective core potential (ECP) with a valence basis set has been extensively used to overcome difficulties. ${ }^{27,29-32}$ Another alternative is to employ relativistic density functional theory (DFT) along with an accurate allelectron basis set. To our knowledge, for the gold clusters, the latter strategy has not been used so far. Consequently, it is important to carry out such calculations with the purpose to compare with the ECP results.

\section{Methodology}

Using the second-order Douglas-Kroll-Hess (DKH2) ${ }^{46-48}$ Hamiltonian as available in Gaussian 09 code, ${ }^{49}$ the B3PW91 functional ${ }^{50,51}$ with the relativistic all-electron basis set of valence double zeta quality plus polarization functions and one diffuse function of d symmetry $(\mathrm{DZP}+1 \mathrm{~d}-\mathrm{DKH})^{52}$ are employed to optimize the ground state geometries of the small gold clusters $\left(\mathrm{Au}_{\mathrm{n}}, \mathrm{n} \leq 8\right)$. Then, the binding energies $(\mathrm{BE})$, vertical ionization potentials (VIP) and electron affinities (VEA), and HOMO-LUMO energy gaps $\left(\mathrm{E}_{\mathrm{g}}\right)$ are evaluated. To compute the static mean dipole polarizabilities $(\bar{\alpha})$ and polarizability anisotropies $(\Delta \alpha)$ of the gold clusters, the augmented TZP-DKH (ATZP-DKH) basis set ${ }^{53}$ is used. As the Gaussian 09 program does not carry out DKH calculations of second hyperpolarizability $(\bar{\gamma})$, it is obtained from the non-relativistic Hamiltonian and the ATZP set. ${ }^{53}$ These basis sets can be found in many different formats at the web site of Grupo de Química Quântica (UFES, Brazil), at Downloads section. ${ }^{54}$ The d exponent added to the DZP-DKH basis set is equal to 0.35702456 . It is known that the B3PW91 functional has had success in describing electronic structures of transition metal clusters (see Assadollahzadeh and Schwerdtfeger, ${ }^{30}$ Jorge et al., ${ }^{40,41}$ and references therein).

The VIP, VEA, $\mathrm{E}_{\mathrm{g}}$, and $\mathrm{BE}$ are defined as:

$$
\begin{aligned}
& \text { VIP }=E(N-1)-E(N) \\
& V E A=E(N)-E(N+1) \\
& E_{g}=E(L U M O)-E(H O M O) \\
& B E=n_{A u}-E_{\text {Aun }}
\end{aligned}
$$

where $E(N)$ is the cluster energy containing $N$ electrons and $\mathrm{n}$ is the number of gold atoms. $\bar{\alpha}, \Delta \alpha$, and $\bar{\gamma}$ are computed as:

$\bar{\alpha}=\left(\alpha_{\mathrm{zz}}+\alpha_{\mathrm{xx}}+\alpha_{\mathrm{yy}}\right) / 3$
$\Delta \alpha=(1 / 2)^{1 / 2}\left[\left(\alpha_{\mathrm{xx}}-\alpha_{\mathrm{yy}}\right)^{2}+\left(\alpha_{\mathrm{yy}}-\alpha_{\mathrm{zz}}\right)^{2}+\left(\alpha_{\mathrm{zz}}-\alpha_{\mathrm{xx}}\right)^{2}\right]^{1 / 2}$
$\bar{\gamma}=(1 / 5)\left(\gamma_{\mathrm{xxxx}}+\gamma_{\mathrm{yyyy}}+\gamma_{\mathrm{zzzz}}+2 \gamma_{\mathrm{xxyy}}+2 \gamma_{\mathrm{yyzz}}+2 \gamma_{\mathrm{zzxx}}\right)$

\section{Results and Discussion}

\section{Structure}

As the geometries of the gold clusters with up to 8 atoms were widely studied earlier and as they are well established in the literature nowadays, the symmetries reported in Taylor et al. ${ }^{28}$ Assadollahzadeh and Schwerdtfeger ${ }^{30}$ and Li et al..$^{31}$ are chosen in this work. Like the copper and silver clusters, the multiplicities of the even- and odd-numbered gold clusters are one and two, respectively.

Table 1 displays the DKH2-B3PW91/DZP+1d-DKH average bond distances $\left(\mathrm{R}_{\mathrm{ave}}\right)$ of the gold clusters, while Figure 1 shows the corresponding optimized geometries. One can note that all studied clusters prefer planar geometries, whereas $\mathrm{Cu}_{7}, \mathrm{Cu}_{8}, \mathrm{Ag}_{7}$, and $\mathrm{Ag}_{8}$ are three-dimension structures. ${ }^{40,41}$ The B3PW91 results ${ }^{30}$ obtained with the more extensive valence basis set (BS) of the Stuttgart small-core relativistic pseudopotential are included in the Table 1 as well.

For $\mathrm{Au}_{2}$, the experimental values ${ }^{55}$ for the bond length $(2.47 \AA)$ and harmonic vibrational frequency $\left(191 \mathrm{~cm}^{-1}\right)$ agree very well with our results of $2.47 \AA$ and $201.38 \mathrm{~cm}^{-1}$, respectively. The bond length reported by Assadollahzadeh and Schwerdtfeger ${ }^{30}$ using the relativistic B3PW91/BS approach overestimates the experimental result by $0.04 \AA$. Going from $\mathrm{Cu}_{2}(2.219 \AA)^{40}$ to $\mathrm{Ag}_{2}(2.535 \AA)^{41}$ there is an enlargement of the bond distance because the electronic shielding effect overcomes the scalar relativistic effect, whereas going from $\mathrm{Ag}_{2}$ to $\mathrm{Au}_{2}$ the opposite occurs. For the vibrational frequency, the DKH-CCSD(T)/PJHN-4f $2 \mathrm{~g} 1 \mathrm{~h}$ model ${ }^{56}$ predicted a value close to ours, $189 \mathrm{~cm}^{-1}$.

For the ground state of $\mathrm{Au}_{3}$, our optimization process gives a triangular structure containing an obtuse angle $\left(100^{\circ}\right)$ and with a $\mathrm{C}_{2 \mathrm{v}}$ symmetry. It is in line with some theoretical results ${ }^{30,31}$ and with experimental EPR spectra. ${ }^{57,58}$ Although a $\mathrm{D}_{3 \mathrm{~h}}$ symmetry has been predicted by some theoretical approaches, ${ }^{59,60}$ it is not possible due to the Jahn-Teller effect, which distort the equilateral triangle. It 
Table 1. The average cluster bond length $\left(\mathrm{R}_{\text {ave }}\right.$ ), binding energy (BE), vertical ionization potential (VIP), vertical electron affinity (VEA), and HOMOLUMO energy gap $\left(\mathrm{E}_{\mathrm{g}}\right)$ calculated for the ground state gold clusters

\begin{tabular}{|c|c|c|c|c|c|c|c|}
\hline \multirow{2}{*}{ Cluster } & \multicolumn{2}{|c|}{$\mathrm{R}_{\text {ave }} / \AA$} & \multirow{2}{*}{$\begin{array}{c}\mathrm{BE} / \mathrm{eV} \\
\mathrm{DZP}+1 \mathrm{~d}-\mathrm{DKH}^{\mathrm{a}}\end{array}$} & \multicolumn{2}{|c|}{$\mathrm{VIP} / \mathrm{eV}$} & \multirow{2}{*}{$\begin{array}{c}\mathrm{VEA} / \mathrm{eV} \\
\mathrm{DZP}+1 \mathrm{~d}-\mathrm{DKH} \mathrm{H}^{\mathrm{a}}\end{array}$} & \multirow{2}{*}{$\frac{\mathrm{E}_{\mathrm{g}} / \mathrm{eV}}{\mathrm{DZP}+1 \mathrm{~d}-\mathrm{DKH}}$} \\
\hline & $\mathrm{DZP}+1 \mathrm{~d}-\mathrm{DKH} \mathrm{H}^{\mathrm{a}}$ & $\mathrm{BS}^{\mathrm{b}}$ & & $\mathrm{DZP}+1 \mathrm{~d}-\mathrm{DKH} \mathrm{H}^{\mathrm{a}}$ & Experimental $^{\mathrm{c}}$ & & \\
\hline $\mathrm{Au}$ & - & - & - & 9.724 & 9.23 & 2.524 & 2.345 \\
\hline $\mathrm{Au}_{2}\left(\mathrm{D}_{\infty \mathrm{h}}\right)$ & 2.470 & 2.519 & 2.533 & 9.981 & 9.50 & 2.270 & 3.246 \\
\hline $\mathrm{Au}_{3}\left(\mathrm{C}_{2 \mathrm{v}}\right)$ & 2.476 & 2.564 & 4.067 & 8.249 & 7.50 & 2.934 & 1.696 \\
\hline $\mathrm{Au}_{4}\left(\mathrm{D}_{2 \mathrm{~h}}\right)$ & 2.616 & 2.613 & 7.187 & 8.379 & 8.60 & 2.731 & 2.185 \\
\hline $\mathrm{Au}_{5}\left(\mathrm{C}_{2 \mathrm{v}}\right)$ & 2.618 & 2.627 & 10.021 & 7.941 & 8.00 & 3.249 & 1.494 \\
\hline $\mathrm{Au}_{6}\left(\mathrm{D}_{3 \mathrm{~h}}\right)$ & 2.629 & 2.633 & 13.824 & 8.392 & 8.80 & 2.424 & 2.937 \\
\hline $\mathrm{Au}_{7}\left(\mathrm{C}_{1}\right)$ & 2.628 & 2.642 & 16.002 & 7.540 & 7.80 & 3.356 & 1.293 \\
\hline $\mathrm{Au}_{8}\left(\mathrm{D}_{4 \mathrm{~h}}\right)$ & 2.595 & 2.625 & 19.451 & 7.737 & 8.65 & 3.125 & 1.844 \\
\hline
\end{tabular}

${ }^{a}$ This work (DKH2-B3PW91). DKH all-electron basis set from Canal Neto and Jorge $;{ }^{52}$ b3PW91 result from Assadollahzadeh and Schwerdtfeger, ${ }^{30}$ using the more extensive valence basis set of the Stuttgart small-core relativistic pseudopotential; 'from Jackschath et al. ${ }^{26}$
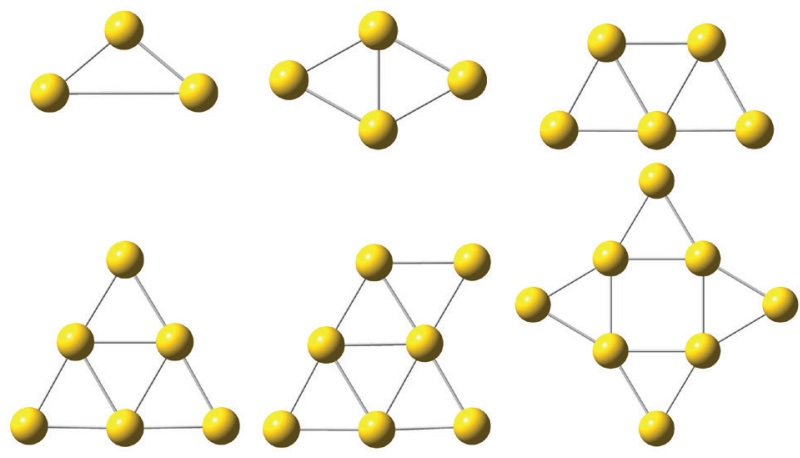

Figure 1. Ground state structures of neutral gold clusters, $\mathrm{Au}_{\mathrm{n}}(\mathrm{n}=3-8)$.

is important to mention here that when compared with $\mathrm{Au}_{3}$, the bond angles of $\mathrm{Cu}_{3}$ and $\mathrm{Ag}_{3}$ are reduced to $66^{\circ} .{ }^{40,41} \mathrm{The}$ DKH2-B3PW91/DZP+1d-DKH bond distance (2.476 ̊) of $\mathrm{Au}_{3}$ is smaller than that obtained from the relativistic B3PW91/BS calculations $(2.564 \AA) .{ }^{30}$ In contrast, for the other gold clusters, the $R_{\text {ave }}$ values calculated with these two models are in excellent agreement (cf. Table 1).

\section{Stability}

The binding energies of the gold clusters are exhibited in Table 1 . They increase monotonically with the cluster size. For $\mathrm{Au}_{2}$ and $\mathrm{Au}_{3}$, the DKH2-B3PW91/DZP+1d-DKH BE (2.533 and $4.067 \mathrm{eV})$ are in good accordance with the experimental data of $2.30^{55}$ and $3.80 \pm 0.13 \mathrm{eV},{ }^{61}$ respectively. A value of $2.19 \mathrm{eV}$ for $\mathrm{Au}_{2}$ was reported by Hess and Kaldor ${ }^{56}$ using a higher level of theory (DKH-CCSD(T)/PJHN-4f2g1h). We have also calculated the binding energy per atom (BE/n), namely: $1.267\left(\mathrm{Au}_{2}\right)$, $1.356\left(\mathrm{Au}_{3}\right), 1.797\left(\mathrm{Au}_{4}\right), 2.004\left(\mathrm{Au}_{5}\right), 2.304\left(\mathrm{Au}_{6}\right)$, $2.286\left(\mathrm{Au}_{7}\right)$, and $2.431\left(\mathrm{Au}_{8}\right) \mathrm{eV}$. Like the binding energy, it increases with the cluster size. Increasing the gold cluster size, the BE/n should approach the bulk cohesive energy
$(3.8 \mathrm{eV}){ }^{62}$ The binding energy per atom of $\mathrm{Au}_{8}$ is about $37 \%$ smaller than this value. To improve our result, larger clusters should be considered. The binding energies per atom for the copper, ${ }^{40}$ silver, ${ }^{41}$ and gold clusters are plotted in Figure 2. One can note that the even-numbered gold cluster curve presents local maximums, indicating that each one of these clusters is more stable than their nearest neighbors. In addition, one can verify that the binding energies per atom of the gold clusters are systematically larger than the corresponding ones of the copper and silver clusters. Once again, this difference can be attributed to the larger scalar relativistic effect on gold clusters.

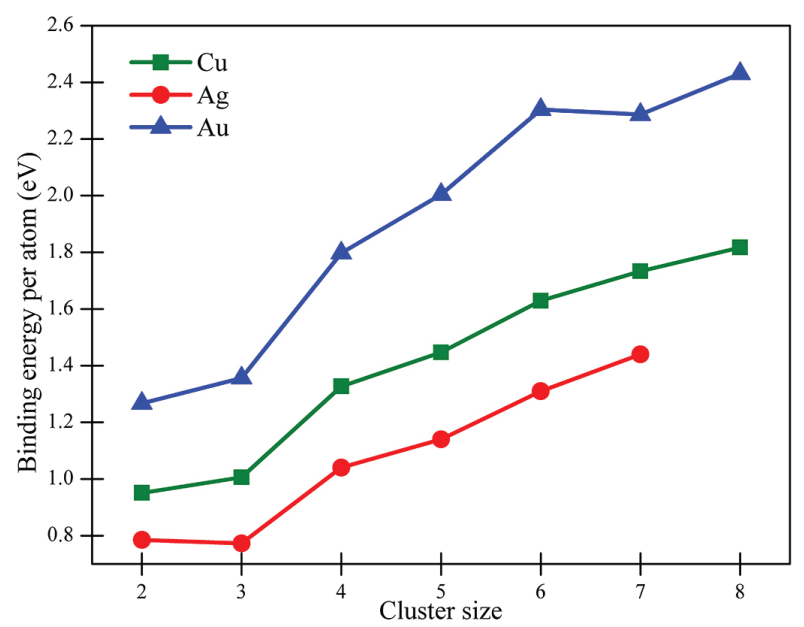

Figure 2. Comparison of binding energies per atom of copper, ${ }^{40}$ silver, ${ }^{41}$ and gold (this work) clusters. In all calculations, the DKH2-B3PW91/XZP-DKH procedure is employed.

The DKH2-B3PW91/DZP+1d-DKH and experimental vertical ionization potentials of the gold clusters are included in Table 1. This property is associated with the trend of the cluster to lose an electron. From this Table 1, one verifies that the theoretical and experimental data 
present odd-even oscillations with the even-numbered clusters having larger VIP. Again, the even-numbered clusters are predicted to be more stable. It should be noted that except for $\mathrm{Au}_{3}$ and $\mathrm{Au}_{8}$, the theoretical values are close to the experimental ones.

Unlike the VIP, the VEA is related with the ability of a cluster to gain an electron. As the HOMO of the odd-numbered gold clusters are partially filled, they have maximum electron affinities, i.e., the odd-even oscillation is exactly the opposite of that of the VIP (cf. Table 1).

The VIP and VEA for the copper, ${ }^{40}$ silver, ${ }^{41}$ and gold clusters are depicted in Figures 3 and 4, respectively. Once more, the relativistic effects are responsible for the larger values of the gold clusters. It is in accordance with the experimental data displayed in Figure 2 of Wesendrup et al. ${ }^{29}$

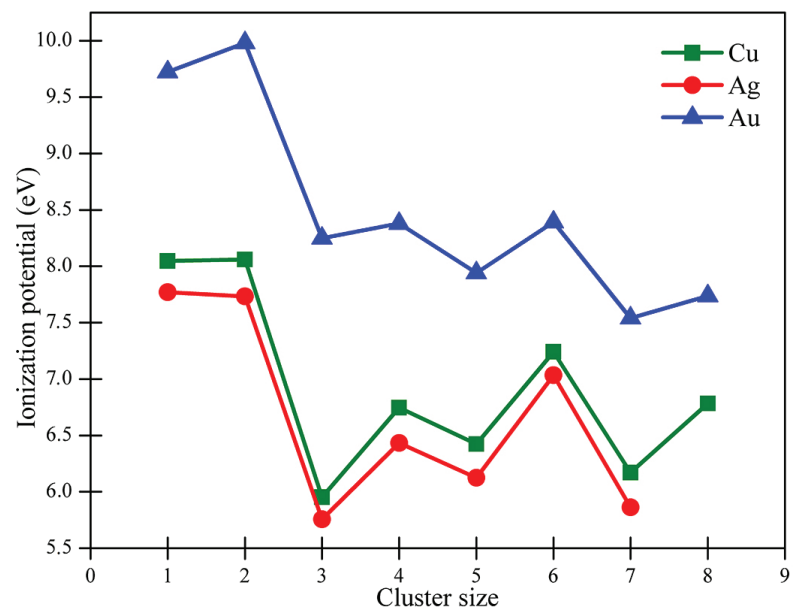

Figure 3. Comparison of ionization potentials of copper, ${ }^{40}$ silver, ${ }^{41}$ and gold (this work) clusters. In all calculations, the DKH2-B3PW91/XZP-DKH procedure is employed.

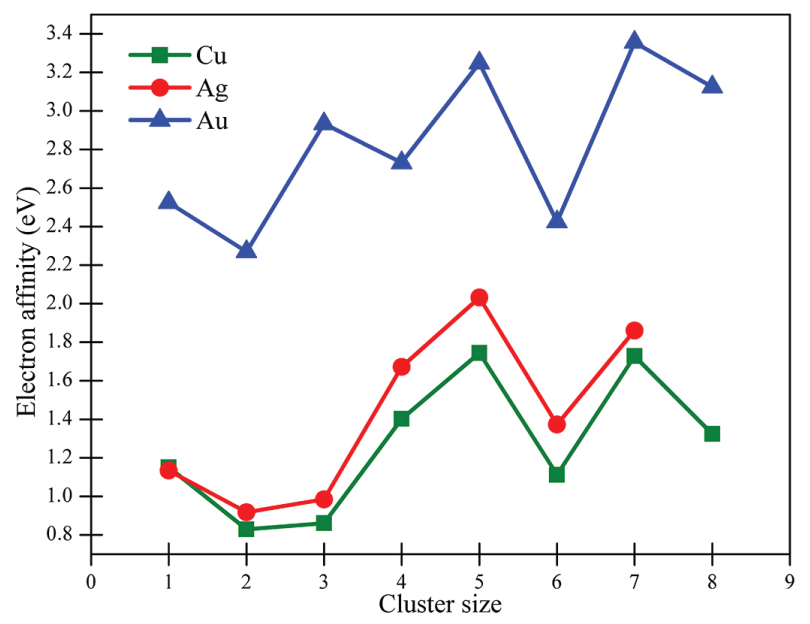

Figure 4. Comparison of electron affinities of copper, ${ }^{40}$ silver, ${ }^{41}$ and gold (this work) clusters. In all calculations, the DKH2-B3PW91/XZP-DKH procedure is employed.

\section{Reactivity}

The DKH2-B3PW91/DZP+1d-DKH HOMO-LUMO energy gaps of the gold clusters are presented in Table 1. This property plays a key role because the chemical activity of a cluster is related with its magnitude. Small HOMO-LUMO gap means that the cluster is chemically active, while large value implies in stability of the cluster. In this work, the HOMO-LUMO energy gaps are used to estimate the reactivities of the gold clusters.

From Table 1, one can note that the HOMO-LUMO gaps produce odd-even oscillations with the maximums and minimums occurring for the even- and odd-numbered clusters, respectively. Consequently, the even-numbered clusters are more stable than the odd ones, which is in accordance with the $\mathrm{BE}$ and VIP results reported in this work. Besides, the smallest value of $\mathrm{E}_{\mathrm{g}}$ is found for $\mathrm{Au}_{7}$, thus, it is the most reactive of the gold clusters included in Table 1. The relativistic B3PW91/BS HOMO-LUMO energy gaps calculated by Assadollahzadeh and Schwerdtfeger ${ }^{30}$ for the gold clusters are in complete agreement with our findings. It should be mentioned here that the larger stability of the even-numbered gold clusters was also predicted by experimental observations..$^{26,28}$

Figure 5 displays the HOMO-LUMO gaps for the copper, ${ }^{40}$ silver, ${ }^{41}$ and gold clusters. The copper and silver clusters present similar oscillating features to that of the gold clusters. Differently of the other properties studied in this work, the HOMO-LUMO energy gaps of the noble metal clusters seem to be equally affected by the relativistic scalar effects.

\section{(Hyper)polarizabilities}

From the optimized geometries of the gold clusters with up to 8 atoms shown in Figure 1, the DKH2-B3PW91/ATZP-DKH static mean dipole polarizability, mean dipole polarizability per atom, and polarizability anisotropy are evaluated and exhibited in Table 2. As the Gaussian 09 program $^{49}$ does not carry out DKH calculation of second hyperpolarizability, the non-relativistic Hamiltonian and basis set (ATZP) are employed.

For $\mathrm{Au}$, an experimental estimate of the mean dipole polarizability (39.1 \pm 9.8 a.u.) was reported by Roos et al. ${ }^{63}$ Our value of 35.45 a.u. is within the experimental uncertainty bars and agrees very well with the result of 36.06 a.u. ${ }^{64}$ computed at a higher level of theory, DKH-CCSD(T)/NpPolMe+(3s3p1d1f ).

As expected, the number of electrons and the mean dipole polarizabilities rise from $\mathrm{Au}$ to $\mathrm{Au}_{8}$. This trend 


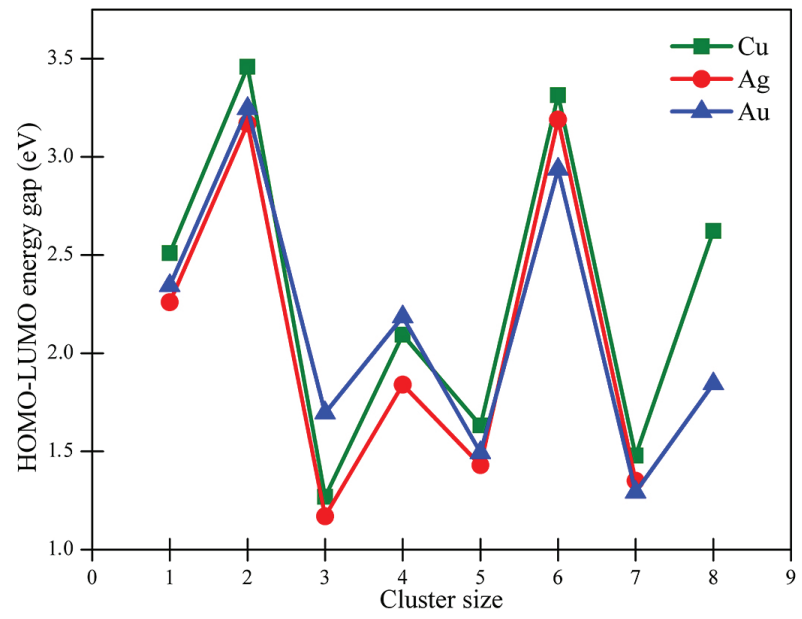

Figure 5. Comparison of HOMO-LUMO energy gaps of copper ${ }^{40}$ silver, ${ }^{41}$ and gold (this work) clusters. In all calculations, the DKH2-B3PW91/XZP-DKH procedure is employed.

Table 2. Static electric mean dipole polarizability $(\bar{\alpha})$, mean dipole polarizability per atom $(\bar{\alpha} / \mathrm{n})$, polarizability anisotropy $(\Delta \alpha)$, and second hyperpolarizability $(\bar{\gamma})$ for the ground state gold clusters

\begin{tabular}{lcccc}
\hline Cluster & \multicolumn{3}{c}{ ATZP-DKH $^{\mathrm{a}}$} & ATZP $^{\mathrm{b}}$ \\
\cline { 2 - 5 } & $\bar{\alpha} /$ a.u. & $\bar{\alpha} / \mathrm{n} /$ a.u. & $\Delta \alpha /$ a.u. & $10^{-3} \times \bar{\gamma} /$ a.u. \\
\hline $\mathrm{Au}$ & 35.45 & 35.45 & 0.00 & 16.48 \\
$\mathrm{Au}_{2}$ & 73.68 & 36.84 & 56.86 & 108.21 \\
$\mathrm{Au}_{3}$ & 130.37 & 43.46 & 122.39 & 297.57 \\
$\mathrm{Au}_{4}$ & 158.75 & 39.69 & 148.69 & 380.26 \\
$\mathrm{Au}_{5}$ & 203.89 & 40.78 & 171.85 & 474.55 \\
$\mathrm{Au}_{6}$ & 233.21 & 38.87 & 172.29 & 463.73 \\
$\mathrm{Au}_{7}$ & 294.90 & 42.13 & 265.11 & 891.17 \\
$\mathrm{Au}_{8}$ & 306.86 & 38.36 & 236.61 & 679.49 \\
\hline
\end{tabular}

${ }^{a}$ This work (DKH2-B3PW91), all-electron basis set from Martins et al. ${ }^{53}$ ${ }^{\mathrm{b}}$ this work (B3PW91), all-electron basis set from Martins et al..$^{53}$

was also observed previously for the copper and silver clusters. ${ }^{40,41}$ When compared with the corresponding results of the lithium and sodium clusters, ${ }^{65}$ the mean dipole polarizabilities of the gold clusters are significantly smaller. It is mainly attributed to the scalar relativistic effects.

For $\mathrm{n} \geq 2$, the mean dipole polarizabilities per atom are characterized by an odd-even oscillation with the maximum values occurring for the odd-numbered clusters. It is exactly the opposite what happens with the HOMO-LUMO energy gaps (cf. Table 1). Clusters with larger $\mathrm{E}_{\mathrm{g}}$ values are more stable, so it is expected that their $\bar{\alpha} / \mathrm{n}$ values to be smaller. Our results confirm the relationship between $\mathrm{E}_{\mathrm{g}}$ and $\bar{\alpha} / \mathrm{n}$. The heptamer, which is predicted to be the most reactive cluster studied in this work, has the second highest value of $\bar{\alpha} / \mathrm{n}$, being only 1.33 a.u. smaller than that of the trimer.

The mean dipole polarizabilities per atom for the copper, silver, and gold clusters with up to six atoms are shown in Figure 6. Now, the $\bar{\alpha} / \mathrm{n}$ values for the gold clusters are among those of the copper and silver clusters. Non-relativistic mean dipole polarizabilities per atom are calculated by our group as well. We verify that the average reductions from the non-relativistic to the relativistic $\bar{\alpha} / \mathrm{n}$ values for the copper, silver, and gold clusters are 1.79, 4.86, and 16.14 a.u., respectively. These results show clearly that it is essential to include scalar relativistic effects on polarizability calculations of gold clusters.

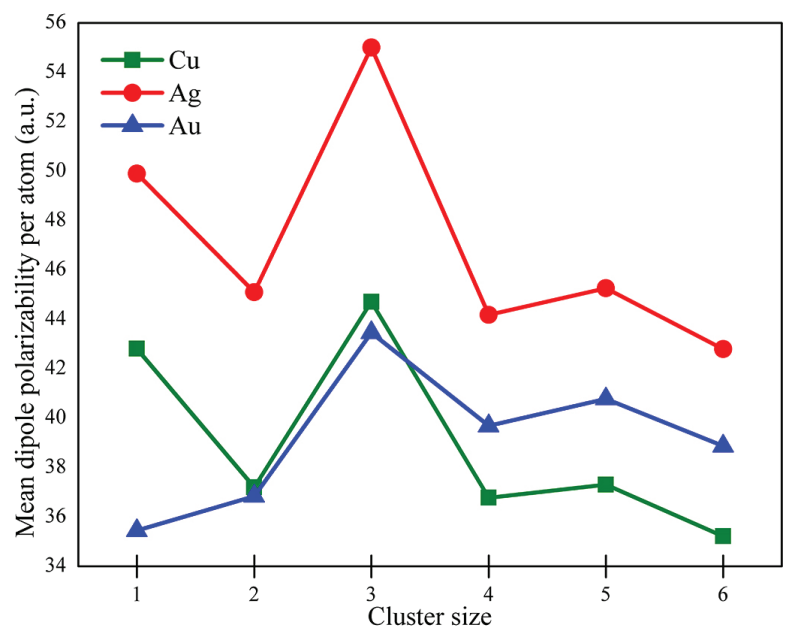

Figure 6. Comparison of mean dipole polarizabilities per atom of copper, silver, and gold clusters. In all calculations, the DKH2-B3PW91/AXZP-DKH procedure is employed.

For planar clusters, it is expected that the polarizability anisotropy increases with the cluster size. As all clusters studied in this work are planar, the DKH2-B3PW91/ATZP-DKH polarizability anisotropies augment from $\mathrm{Au}$ to $\mathrm{Au}_{7}$ (cf. Table 2). A reduction of $\Delta \alpha$ going from $\mathrm{Au}_{7}$ to $\mathrm{Au}_{8}$ can be explained through an exchange of the point group $\left(\mathrm{C}_{1} \rightarrow \mathrm{D}_{4 \mathrm{~h}}\right)$, doing the octamer electronic structure more compact. For the copper and silver clusters, it was observed a sudden fall of $\Delta \alpha$ from the heptamer because of its three-dimensional structure.

In Table 2, the B3PW91/ATZP second hyperpolarizabilities for the gold clusters are presented. They augment from $\mathrm{Au}$ to $\mathrm{Au}_{5}$, while from $\mathrm{Au}_{6}$ appears an odd-even oscillation due to symmetry changes of the clusters. The second hyperpolarizability of $\mathrm{Au}_{7}$ is considerably larger than the other ones because it is the only cluster belonging to the $\mathrm{C}_{1}$ point group. We do not have any knowledge about second hyperpolarizability published previously for any gold cluster. So, our results will be compared with those calculated for the copper and silver clusters at the same level of theory. ${ }^{40,41}$ Except for the monomer, the second hyperpolarizabilities of the silver and gold clusters are in general of the same magnitudes and they are larger than those of the copper clusters. 


\section{Conclusions}

In this work, optimized geometries, binding energies, vertical ionization potentials and electron affinities, HOMO-LUMO energy gaps, and polarizabilities of gold clusters with up to 8 atoms are evaluated using the DKH2 Hamiltonian and relativistic all-electron basis sets.

With exception of $\mathrm{Au}_{3}$, the agreement between the corresponding DKH2-B3PW91/DZP+1d-DKH and relativistic B3PW91/BS ${ }^{30}$ average bond lengths is good. For $\mathrm{Au}_{2}$, our result of $2.47 \AA$ is exactly equal to the experimental value. ${ }^{55}$ For $\mathrm{Au}_{3}$, an obtuse angle is predicted in this work. It is in line with theoretical ${ }^{30,31}$ and experimental data ${ }^{57,58}$ reported in the literature.

For $\mathrm{Au}_{2}$ and $\mathrm{Au}_{3}$, the DKH2-B3PW91/DZP+1d-DKH binding energies are close to the experimental values. ${ }^{55,61}$ With the exceptions of $\mathrm{Au}_{3}$ and $\mathrm{Au}_{8}$, the DKH2-B3PW91/DZP+1d-DKH ionization potentials are in good agreement with the experimental values. The binding energies per atom and the vertical ionization potentials present oscillating characteristics indicating that the evennumbered clusters are more stable. The oscillating feature of the vertical electron affinities is exactly the opposite of that found for the vertical ionization potentials. This finding is in accordance with the chemical intuition.

The odd-even oscillation of the HOMO-LUMO energy gaps is like those of the BE and VIP, i.e., it confirms the larger stability of the even-numbered clusters in relation to its closest neighbors. In contrast, the $\mathrm{Au}_{7}$ cluster is predicted to be the most reactive of all.

Odd-even oscillations with maximum values occurring for the odd-numbered clusters characterize the static mean dipole polarizabilities per atom of the gold clusters. High reactivity is associated with high mean dipole polarizability per atom. It should be noted that the mean dipole polarizability per atom of $\mathrm{Au}_{7}$ confirms this expectancy. As expected for planar clusters, the polarizability anisotropies increase from $\mathrm{Au}_{2}$ to $\mathrm{Au}_{7}$. There is a reduction from $\mathrm{Au}_{7}$ to $\mathrm{Au}_{8}$ due to a change in the symmetry. The electronic structure of the $\mathrm{Au}_{8}$ cluster is more compact than that of $\mathrm{Au}_{7}$. It is the first time that the second hyperpolarizabilities of the gold clusters $\left(\mathrm{Au}_{\mathrm{n}}, \mathrm{n} \leq 8\right)$ are reported in the literature. From $\mathrm{Au}$ to $\mathrm{Au}_{5}$ there is an enlargement, whereas from $\mathrm{Au}_{6}$ an odd-even oscillation appears, being the second hyperpolarizability of $\mathrm{Au}_{7}\left(\mathrm{C}_{1}\right.$ point group) considerably larger than the other clusters.

The so expected large scalar relativistic effects on BE/n, VIP, and VEA calculations of the gold clusters when compared with those of the copper and silver clusters are confirmed in this work using the DKH2-B3PW91/XZP-DKH model.

\section{Acknowledgments}

We would like to acknowledge the financial support of CNPq, CAPES, and FAPES (Brazilian agencies).

\section{References}

1. Jellinek, J.; Theory of Atomic and Molecular Clusters; Springer: Berlin, 1999.

2. Ueno, L. T.; Ornellas, F. R.; J. Braz. Chem. Soc. 2001, 12, 99.

3. Koutecky, V. B.; Veyret, V.; Mitric, R.; J. Chem. Phys. 2001, 115,10450 .

4. Martínez, A.; J. Braz. Chem. Soc. 2005, 16, 337.

5. Pereiro, M.; Baldomir, D.; Phys. Rev. A 2007, 75, 033202.

6. Böyükata, M.; Belchior, J. C.; J. Braz. Chem. Soc. 2008, 19, 884.

7. Li, W. Y.; Chen, F. Y.; Chin. Phys. B 2014, 23, 117103.

8. Zhang, J. F.; Zhang, M.; Zhao, Y. W.; Zhang, H. Y.; Zhao, L. N.; Luo, Y. H.; Chin. Phys. B 2015, 24, 067101.

9. Chang, Y.; Li, W.; Jiang, Y.; Phys. Lett. A 2012, 376, 2314.

10. Lv, J.; Zhang, J. Y.; Liang, R. R.; Wu, H. S.; Chin. Phys. B 2016, $25,063103$.

11. Xing, X.; Hermann, A.; Kuang, X.; Ju, M.; Lu, C.; Jin, Y.; Xia, X.; Maroulis, G.; Sci. Rep. 2016, 6, 19656.

12. Rabin, I.; Jackschath, C.; Schulze, W.; Z. Phys. D: At., Mol. Clusters 1991, 19, 153.

13. Jackschath, C.; Rabin, I.; Schulze, W.; Z. Phys. D: At., Mol. Clusters 1992, 22, 517.

14. Spasov, V. A.; Lee, T. H.; Maberry, J. P.; Ervin, K. M.; J. Chem. Phys. 1999, 110, 5208.

15. Shi, Y.; Spasov, V. A.; Ervin, K. M.; J. Chem. Phys. 1999, 111, 938.

16. Ho, J.; Erwin, K. M.; Lineberger, W. C.; J. Chem. Phys. 1990 , 93, 6987.

17. Alammeddin, G.; Hunter, J.; Cameron, D.; Kappes, M. M.; Chem. Phys. Lett. 1992, 192, 122.

18. Handschuh, H.; Cha, C. Y.; Bechthold, P. S.; Ganteför, G.; Eberhardt, W.; J. Chem. Phys. 1995, 102, 6406.

19. Khan, S. A.; Senapati, D.; Senapati, T.; Bonifassi, P.; Fan, Z.; Singh, A. K.; Neeley, A.; Hill, G.; Ray, P. C.; Chem. Phys. Lett. 2011, 512, 92.

20. Schooss, D.; Gilb, S.; Kaller, J.; Kappes, M.; Furche, F.; Kohn, A.; May, K.; Ahlrichs, R.; J. Chem. Phys. 2000, 113, 5361.

21. Hakkinen, H.; Yoon, B.; Landman, U.; Li, X.; Zhai, H.-J.; Wang, L.-S.; J. Phys. Chem. A 2003, 107, 6168.

22. Vogel, M.; Hansen, K.; Herlert, A.; Schweikhard, L.; Eur. Phys. J. D 2001, 21, 163.

23. Liu, Z. F.; Yim, W. L.; Tse, J. S.; Hafner, J.; Eur. Phys. J. D 2000, 10, 105.

24. Zhao, J.; Yang, J.; Hou, J. G.; Phys. Rev. B 2003, 67, 085404. 
25. Weis, P.; Bierweiler, T.; Gilb, S.; Kappes, M. M.; Chem. Phys. Lett. 2002, 355, 355.

26. Jackschath, C.; Rabin, I.; Schulze, W.; Ber. Bunsenges. Phys. Chem. 1992, 96, 1200.

27. Balasubramanian, K.; Das, K. K.; Chem. Phys. Lett. 1991, 186, 577.

28. Taylor, K. J.; Pettiette-Hall, C. L.; Cheshnovsky, O.; Smalley, R. E.; J. Chem. Phys. 1992, 96, 3319.

29. Wesendrup, R.; Hunt, T.; Schwerdtfeger, P.; J. Chem. Phys. 2000, 112, 9356.

30. Assadollahzadeh, B.; Schwerdtfeger, P.; J. Chem. Phys. 2009, 131, 064306.

31. Li, X.-B.; Wang, H.-Y.; Yang, X.-D.; Zhu, Z.-H.; J. Chem. Phys. 2007, 126, 084505.

32. Zanti, G.; Peeters, D.; Theor. Chem. Acc. 2013, 132, 1300.

33. Van Lenthe, E.; van der Avoird, A.; Wormer, P. E. S.; J. Chem. Phys. 1998, 108, 4783.

34. Hakkinen, H.; Moseler, M.; Landman, U.; Phys. Rev. Lett. 2002, $89,033401$.

35. Powers, D. E.; Hansen, S. G.; Geusic, M. E.; Michalopoulos, D. L.; Smalley, R. E.; J. Chem. Phys. 1983, 78, 2866.

36. Knickelbein, M. B.; Chem. Phys. Lett. 1992, 192, 129.

37. James, A. M.; Lemire, G. W.; Langridge-Smith, P. R. R.; Chem. Phys. Lett. 1994, 227, 503.

38. Rabin, I.; Jackschath, C.; Schulze, W.; Z. Phys. D: At., Mol. Clusters 1991, 19, 153.

39. Jackschath, C.; Rabin, I.; Schulze, W.; Z. Phys. D: At., Mol. Clusters 1992, 22, 517.

40. Jorge, F. E.; Ferreira, I. B.; Soprani, D. D.; Gomes, T.; J. Braz. Chem. Soc. 2016, 27, 127.

41. Jorge, F. E.; de Macedo, L. G. M.; Chin. Phys. B 2016, 25, 123102.

42. Haruta, M.; CATTECH 2002, 6, 102.

43. Haruta, M.; Gold Bull. 2004, 37, 27.

44. Haruta, M.; Yamada, N.; Kobayashi, T.; Iijima, S.; J. Catal. 1989, 115, 301.

45. Schwerdtfeger, P.; Angew Chem., Int. Ed. 2003, 42, 1892.

46. Douglas, M.; Kroll, N. M.; Ann. Phys. 1974, 82, 89.

47. Hess, B. A.; Phys. Rev. A: At., Mol., Opt. Phys. 1985, $32,756$.

48. Hess, B. A.; Phys. Rev. A: At., Mol., Opt. Phys. 1986, 33, 3742.

49. Frisch, M. J.; Trucks, G. W.; Schlegel, H. B.; Scuseria, G. E.; Robb, M. A.; Cheeseman, J. R.; Scalmani, G.; Barone, V.; Mennucci, B.; Petersson, G. A.; Nakatsuji, H.; Caricato, M.;
Li, X.; Hratchian, H. P.; Izmaylov, A. F.; Bloino, J.; Zheng, G.; Sonnenberg, J. L.; Hada, M.; Ehara, M.; Toyota, K.; Fukuda, R.; Hasegawa, J.; Ishida, M.; Nakajima, T.; Honda, Y.; Kitao, O.; Nakai, H.; Vreven, T.; Montgomery Jr., J. A.; Peralta, J. E.; Ogliaro, F.; Bearpark, M.; Heyd, J. J.; Brothers, E.; Kudin, K. N.; Staroverov, V. N.; Kobayashi, R.; Normand, J.; Raghavachari, K.; Rendell, A.; Burant, J. C.; Iyengar, S. S.; Tomasi, J.; Cossi, M.; Rega, N.; Millam, J. M.; Klene, M.; Knox, J. E.; Cross, J. B.; Bakken, V.; Adamo, C.; Jaramillo, J.; Gomperts, R.; Stratmann, R. E.; Yazyev, O.; Austin, A. J.; Cammi, R.; Pomelli, C.; Ochterski, J. W.; Martin, R. L.; Morokuma, K.; Zakrzewski, V. G.; Voth, G. A.; Salvador, P.; Dannenberg, J. J.; Dapprich, S.; Daniels, A. D.; Farkas, Ö.; Foresman, J. B.; Ortiz, J. V.; Cioslowski, J.; Fox, D. J.; Gaussian 09, Revision A.02, Gaussian Inc.: Wallingford, CT, 2009.

50. Becke, A. D.; J. Chem. Phys. 1993, 98, 5648.

51. Perdew, J. P.; Wang, W. R.; Phys. Rev. B: Condens. Matter Mater. Phys. 1992, 45, 13244.

52. Canal Neto, A.; Jorge, F. E.; Chem. Phys. Lett. 2013, 582, 158.

53. Martins, L. S. C.; Jorge, F. E.; Machado, S. F.; Mol. Phys. 2015, 113,3578 .

54. http://qcgv.ufes.br/, accessed in November 2017.

55. Huber, K. P.; Herzberg, G.; Constants of Diatomic Molecules; Van Nostrand Reinhold: New York, 1979.

56. Hess, B. H.; Kaldor, U.; J. Chem. Phys. 2000, 112, 1809.

57. Howard, J. A.; Sutcliffe, R.; Mille, B.; J. Chem. Soc. Chem. Commun. 1983, 1499.

58. Howard, J. A.; Sutcliffe, R.; Mille, B.; Surf. Sci. 1985, 156, 214.

59. Grönbeck, H.; Andreoni, W.; Chem. Phys. 2000, 262, 1.

60. Wang, J.; Wan, G.; Zhao, J.; Phys. Rev. B 2002, 66, 035418.

61. Hilpert, K.; Gingerich, K. A.; Ber. Bunsenges. Phys. Chem. 1980, 84, 739 .

62. Kittel, C.; Introduction to Solid State Physics, $3^{\text {rd }}$ ed.; Wiley: New York, 1976.

63. Roos, B. O.; Lindh, R.; Malmqvist, P.-A.; Veryazov, V.; Widmark, P.-O.; J. Phys. Chem. A 2005, 109, 6575.

64. Neogrády, P.; Kellö, V.; Urban, M.; Sadlej, A. J.; Int. J. Quantum Chem. 1997, 63, 557.

65. de Souza, F. A. L.; Jorge, F. E.; J. Braz. Chem. Soc. 2013, 24, 1357.

Submitted: September 20, 2017 Published online: November 22, 2017 